\title{
THE PATTERN PRACTICE OF MEANINGS
}

\author{
Edward Anthony \\ University of Michigan
}

The teacher who concentrates on vocabulary has three important duties to discharge. First, he must choose items useful to his students. Second, he must adequately present and explain these items. Third, he must practice these items until their use becomes automatically attached to the experience which they symbolize. It is this third duty which is sometimes neglected, and which will occupy us here. We shall therefore assume that items have been chosen, presented, and explained.

An instructor in driving can quickly explain the mechanism of an auto, the successive operations necessary to set it in motion, turn it, and stop it. His student, however, cannot therefore immediately enter a car and drive surely and easily down the street. A certain amount of gear-clashing, jerking, and sudden stopping is inevitable at first. Only constant practice will cure these faults. A teacher of language likewise cannot expect his student to use words with facility if they have been merely presented. A certain amount of verbal gear-clashing, jerking, and sudden stopping is inevitable. The student must use the items again and again before they are his.

There are several frequently-used techniques for practicing vocabulary items, some useful and practical. Perhaps the most frequent method of practice is that of requiring a sentence from each student using the item in question. Aside from the monotony which often results from such practice, there is a real danger that the students understanding of the item is incomplete, although the example sentence is right. If, for example, a sentence using actually is elicited from the student, a sentence from a native Spanish speaker might be: Is Mr. Brown actually a teacher? This is, of course, an acceptable English sentence. But, in all probability, the student does not mean what he is saying. The word actualmente in Spanish has a time sense that the cognate actually lacks in English. In order for the teacher to be certain that the student understands, he must require the student to construct a sentence which emphasizes the contrast between actualmente and actually, such as; Was $\mathrm{Mr}$. 
EDWARD ANTHONY

Brown actually a teacher? This is an impossible sentence in Spanish with actualmente.

One cannot, of course, suppose that examples freely given will contain elements that will force the meaning to view. Some sort of closely controlled exercises must be evolved. Our first principles for these exercises are taken from grammar pattern practice. Dr. Robert Lado has said, "Pattern practice - completely oral - is one such technique. It consists paradoxically in the conscious substitution of some element other than the chief element being taught so that primary attention is drawn away from it while the entire pattern is repeated. The instructor presents the pattern orally while the students repeat the complete pattern including the substitution." ings has, as an additional principle, that clear-cut contrasts of lexical meaning must be forced to view and emphasized in such a manner that student errors are immediately apparent and therefore immediately correctable.

Now, following these precepts, let us first suppose that the general area in which we are operating is Food and Meals. Let us further assume that the following items have already been presented and explained, and are now to be practiced.

I. Items explained and practiced in previous work.

a. The subject pronouns.

b. The word eat, the third person singular -5

II. Items explained in the present lesson, to be practiced here.

a. at six, seven, eight, etc.

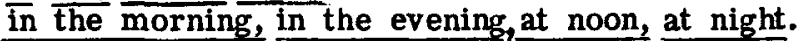

b. breakfast, lunch, dinner, supper.

We begin, then, with the familiar, with the already practiced and learned items, in the frame he eats. We add one of the new expressions from List II a above. This last does not vary. However, we may vary the subject, since the subject pronouns have already been established. The exercise can be presented orally thus:

Teacher; He eats in the evening.

Student A: He eats in the evening.

Teacher: She.

Student B: She eats in the evening.

etc.

${ }^{1}$ Language Learning Volume I, Number 1, p. 25. 
THE PATTERN PRACTICE OF MEANINGS

After this has been practiced, we may change in the evening to another expression in $\mathrm{Il}$ and continue the practice.

Having practiced the list in IIa, we turn now to the list of meals in $\mathrm{Ib}$. and proceed in a similar manner: i.e.: Teacher: He eats breakfast.

Student A: He eats breakfast.

Teacher: She.

Student B: She eats breakfast.

After this has been practiced, we may change breakfast to another expression in IIb and continue the practice.

Up to this point, we have not forced the student to understand what he is saying. But now, after having practiced each individual part of the sentence, we put them together and vary any part of the sentence. Let us take $\mathrm{He}$ eats breakfast at seven in the morning, as a basic frame. The teacher then varies any of the three underlined expressions. This has the advantage of forcing the student to understand the contrasts of meaning, for if he makes an error, it becomes immediately evident.

Teacher: He eats breakfast at seven in the morning.

Student A: He eats breakfast at seven in the morning.

Teacher: In the evening.

Student B: He eats DINNER at seven in the evening.

The student must vary two parts of the basic sentence. In the evening is not compatible with breakfast, so the student must substitute either supper or dinner, and can do so only If he understands the meanings of both the phrases supplied him by the teacher and the word which he himself supplies.

If contrasts of lexical meaning are kept constantly in focus, and if these are practiced by meaningful repetition of the type suggested above, some fluency in the use of lexical forms should be attained by the student. 